\title{
Debt and Growth: Is There a Constant Tipping Point?*
}

\author{
Lixiong Yang ${ }^{\dagger}$
}

School of Management, Lanzhou University, Lanzhou, China.

E-mail address: ylx@lzu.edu.cn

\author{
Jen-Je Su
}

Department of Accounting Finance and Economics, Griffith University, Brisbane, Australia.

E-mail address: j.su@griffith.edu.au

\begin{abstract}
This paper highlights the crucial role of a time-varying threshold effect of public debt on economic growth. Our contribution is two-fold. First, we extend the constant-threshold regression kink model of Hansen (2017) by allowing for a time-varying, state-dependent threshold. Second, we apply our model to investigate the effect of debt on growth, using data from the U.S. over the period of 1791-2009. Our empirical results clearly support a nonlinear debt-threshold effect and the threshold is time-varying and state-dependent.
\end{abstract}

Keywords : Debt; Growth; Testing; Time-varying threshold.

JEL classification : C13, C22, C51, E62.

${ }^{*}$ The first author thanks the support of the Fundamental Research Funds for the Central Universities in China (18LZUJBWZY010).

${ }^{\dagger}$ Corresponding author: Lixiong Yang. School of Management, Lanzhou University, 222 South Tianshui Road, Lanzhou 730000, China. E-mail address: ylx@lzu.edu.cn. Phone: +86 13669327501 


\title{
Debt and Growth: Is There a Constant Tipping Point?
}

\begin{abstract}
This paper highlights the crucial role of a time-varying threshold effect of public debt on economic growth. Our contribution is two-fold. First, we extend the constant-threshold regression kink model of Hansen (2017) by allowing for a time-varying, state-dependent threshold. Second, we apply our model to investigate the effect of debt on growth, using data from the U.S. over the period of 1791-2009. Our empirical results clearly support a nonlinear debt-threshold effect and the threshold is time-varying and state-dependent.
\end{abstract}

Keywords : Debt; Growth; Testing; Time-varying threshold.

JEL classification : C13, C22, C51, E62.

\section{Introduction}

Starting from the seminal work of Reinhart and Rogoff (2010), the linkage between public debt and economic growth has once again attracted much attention among academics and policymakers in recent years. According to Reinhart and Rogoff (2010), while the link between debt and growth seems relatively weak at "normal" debt levels, debt is notably associated with slow growth when exceeding $90 \%$ of GDP. The finding of Reinhart and Rogoff (2010) has gained support by several succeeding papers, including Cecchetti et al. (2011), Checherita-Westphal and Rother (2012) and Baum et al. (2013). However, the issue regarding whether there exists a debt threshold around $90 \%$ is far from settled. For example, Herndon et al. (2014) find that growth at the debt-to-GDP ratio over $90 \%$ is not much different from when the ratio is lower and Lee et al. (2017) reach a debt threshold not around 90\% but around 30\%. See also Egert (2015), Eberhardt and Presbitero (2015) and Chudik et al. (2017) for results against a universally applicable threshold effect. ${ }^{1}$

More recently, apart from the panel-data approach commonly employed in the debtgrowth studies (including all the aforementioned papers), Hansen (2017) adds to the relevant literature via a novel time-series approach. Specially, Hansen (2017) develops a regression kink (RK) model with an unknown constant threshold (kink) to investigate the impact of debt on growth. The RK model is an extension of the widely applied regression discontinuity (RD) model (Card et al., 2017). Contrasting to the RD model, which estimates a "jump" in the outcome associated with a discontinuous jump of a

\footnotetext{
${ }^{1}$ For a thorough review, please see Panizza and Presbistero (2013).
} 
policy variable, the RK model estimates a "kink" in the outcome associated with a continuous policy variable when the variable has a kink. Hence, in the RK model, the regression function is continuous but the slope has a discontinuity at a threshold point ("kink"). Applying the RK model to long-span US time-series data over the period of 1791 to 2009, Hansen (2017) finds a growth slowdown when the debt/GDP ratio surpasses a threshold around 43-44\%; however, the threshold effect is not significant when a formal test is applied. ${ }^{2}$

Given that the finding of Hansen (2017) is based on data covering a prolonged period (more than two centuries), one might concern whether assuming a constant threshold is practical. Moreover, suppose that the threshold is time-varying but being treated as a constant, it is hard to know if the modelling outcomes are still valid. ${ }^{3}$ Allowing for a more flexible, possibly state-dependent, threshold is also called for owning to the observation of Dueker et al. (2013) that "usually high/low values of an economic variable may sometimes be best thought of in relative terms". "For example, the level of public debt may be best regarded as high or low not in absolute terms but relative to pertinent macroeconomic variables (covariates) that shape the state of an economy. As macroeconomic variables are typically time-varying, the associated assessing reference (threshold) is unlikely to be constant. Accordingly, the same level of debt-to-GDP ratio may be regarded as high under a certain condition but only moderate under other conditions.

In this paper, we aim to contribute to the debt-growth literature by addressing the vital role of a time-varying, state-dependent tipping point (threshold). To this end, we develop a modified RK model that extends the Hansen's RK model by allowing for a state-dependent threshold, which is treated as a function of informative covariates. Following Hansen (2017), we illustrate a standard approach in estimating the model and introduce two testing procedures for distinguishing between linear and threshold models and between constant and time-varying thresholds, respectively. We apply the proposed model to the data used in Hansen (2017) with a set of macroeconomic covariates to form the threshold function. Overall, our empirical results lean toward supporting the existence of a tipping point (threshold), above which debt becomes notably harmful

\footnotetext{
${ }^{2}$ See Lin (2014) for a similar finding when a very different empirical framework is considered.

${ }^{3}$ In the Appendix, our Monte Carlo simulations show that overlooking the possible nature of a threshold would lead to seriously biased estimates. Furthermore, the direction and the magnitude of the bias depend on the setting of the involving time-varying threshold parameters.

${ }^{4}$ To the best of our knowledge, Dueker et al. (2013) is the only paper that employs a varying threshold. Dueker et al. (2013) propose a smooth transition autoregressive (STAR) model with a time-varying/state-dependent threshold and apply the model to the dynamics of U.S. short-term interest rates.
} 
for growth. Besides, our results show that, in general, a time-varying/state-dependent threshold is more suitable than a constant one.

Apparently, identifying debt-threshold determinants is challenging because the relevant literature is scant and indirect. In this paper, three possible covariates - growth, inflation, and the past debt/GDP ratio - are attempted. The choice of growth may be justified on the ground that public debt tends to be self-financed when growth is high. With regard to inflation, according to Cochrane (2011a), "The key reason serious inflation often accompanies economic difficulties is straightforward: Inflation is a form of sovereign default"; therefore, inflation may deteriorate the impact of debt to growth. However, default (via inflation) implies reducing the real burden and so high inflation is likely to ease negative growth effects of debt. Given these two opposing effects of inflation, the overall effect of inflation on debt-threshold is unclear. ${ }^{5}$ Finally, considering the past debt/GDP ratio is based on the presumption that an economic variable is often viewed as high or low relative to its own recent past values. When public debt lingers at a certain level for a while, the perceived "normal" debt level (threshold) might shift toward to that level particularly, if other macroeconomic conditions remain stable.

The remainder of this article is organized as follows. Section 2 introduces regression kink (RK) models with time-varying and state-dependent thresholds, describes least squares estimation of the model parameters, and discusses test statistics for threshold effect and threshold constancy. Sections 3 presents the main empirical results. Section 4 provides the results of robustness check, and Section 5 concludes.

\section{The Methodology}

In this section, we first extend the regression kink (RK) model of Hansen (2017) by allowing for a time-varying, state-dependent threshold. We then illustrate the estimation procedure and propose test statistics for threshold effect and threshold constancy.

\subsection{Regression Kink with a State-Dependent Threshold}

Let $y_{t}$ be the real GDP growth rate in year $t, x_{t}$ be the debt-to-GDP percentage from the previous year, and $\boldsymbol{z}_{t}$ be a set of control variables. Our regression kink model with

\footnotetext{
${ }^{5}$ We thank the anonymous referee to raise this point to us. For further discussions on the relation between debt and inflation, please see Cochrane (2011a, b).
} 
a state-dependent threshold can be represented by

$$
y_{t}=\beta_{1}\left(x_{t}-\gamma_{t}\right)_{-}+\beta_{2}\left(x_{t}-\gamma_{t}\right)_{+}+\boldsymbol{\beta}_{3}^{\prime} \boldsymbol{z}_{t}+e_{t}, t=1,2, \ldots, T
$$

where $y_{t}, x_{t}$ and $e_{t}$ are scalars, and $\boldsymbol{z}_{t}$ is a $l$-dimensional vector that includes an intercept. $\left(x_{t}-\gamma_{t}\right)_{-}=\min \left[x_{t}-\gamma_{t}, 0\right]$ and $\left(x_{t}-\gamma_{t}\right)_{+}=\max \left[x_{t}-\gamma_{t}, 0\right]$ denote the negative part and positive part of $x_{t}-\gamma_{t}$, respectively. $\gamma_{t}$ is a time-varying threshold (tipping point), which is specified as a function of informative covariates, e.g., a linear combination of the elements of a $k$-dimensional vector $\boldsymbol{q}_{t}=\left(q_{1, t}, \ldots, q_{k, t}\right)^{\prime}$ of observable exogenous or predetermined variables

$$
\gamma_{t}=\gamma_{0}+\gamma_{1}^{\prime} \boldsymbol{q}_{t}
$$

where $\gamma_{0}$ represents an unknown threshold intercept, $\gamma_{1}=\left(\gamma_{11}, \ldots, \gamma_{1 k}\right)^{\prime}$ is a vector of unknown slope parameters.

The model defined by (1) and (2) can be referred to as a modified RK model with a time-varying/state-dependent threshold. Clearly, the Hansen's RK model with an unknown constant threshold is a special case of our model when $\gamma_{1}=0$.

\section{$2.2 \quad$ Estimation Procedure}

For convenience, we rewrite the model in a more compacted form. Define $\boldsymbol{\beta}=$ $\left[\beta_{1}, \beta_{2}, \boldsymbol{\beta}_{3}^{\prime}\right]^{\prime}, \boldsymbol{x}_{t}\left(\gamma_{0}, \gamma_{1}^{\prime}\right)=\left[\left(x_{t}-\gamma_{t}\right)_{-},\left(x_{t}-\gamma_{t}\right)_{+}, \boldsymbol{z}_{t}^{\prime}\right]^{\prime}$. Then model (1) can be rewritten as $y_{t}=\boldsymbol{\beta}^{\prime} \boldsymbol{x}_{t}\left(\gamma_{0}, \gamma_{1}^{\prime}\right)+e_{t}$. The least-square criterion is

$$
S S R_{T}\left(\boldsymbol{\beta}, \gamma_{0}, \gamma_{1}^{\prime}\right)=\sum_{t=1}^{T}\left(y_{t}-\boldsymbol{\beta}^{\prime} \boldsymbol{x}_{t}\left(\gamma_{0}, \boldsymbol{\gamma}_{1}^{\prime}\right)\right)^{2}
$$

For any fixed $\gamma_{0}$ and $\gamma_{1}^{\prime}$, we have the following least-square estimation

$$
y_{t}=\hat{\boldsymbol{\beta}}^{\prime}\left(\gamma_{0}, \gamma_{1}^{\prime}\right) \boldsymbol{x}_{t}\left(\gamma_{0}, \gamma_{1}^{\prime}\right)+\hat{e}_{t}\left(\gamma_{0}, \gamma_{1}^{\prime}\right)
$$

where $\hat{\boldsymbol{\beta}}\left(\gamma_{0}, \gamma_{1}^{\prime}\right)$ is given by $\hat{\boldsymbol{\beta}}\left(\gamma_{0}, \boldsymbol{\gamma}_{1}^{\prime}\right)=\left[\sum_{t=1}^{T} \boldsymbol{x}_{t}\left(\gamma_{0}, \gamma_{1}^{\prime}\right) \boldsymbol{x}_{t}^{\prime}\left(\gamma_{0}, \gamma_{1}^{\prime}\right)\right]^{-1}\left[\sum_{t=1}^{T} \boldsymbol{x}_{t}\left(\gamma_{0}, \boldsymbol{\gamma}_{1}^{\prime}\right) y_{t}\right]$. Accordingly, define $\widetilde{S S R}+\left(\gamma_{0}, \gamma_{1}^{\prime}\right)=\sum_{t=1}^{T}\left(y_{t}-\hat{\boldsymbol{\beta}}^{\prime}\left(\gamma_{0}, \boldsymbol{\gamma}_{1}^{\prime}\right) \boldsymbol{x}_{t}\left(\gamma_{0}, \boldsymbol{\gamma}_{1}^{\prime}\right)\right)^{2}$. Then the parameters of the time-varying threshold can be given as

$$
\left(\hat{\gamma}_{0}, \hat{\gamma}_{1}^{\prime}\right)=\underset{\left(\gamma_{0}, \gamma_{1}^{\prime}\right) \in \Gamma_{0} \times \Gamma_{1}}{\arg \min } \widetilde{S S R_{T}}\left(\gamma_{0}, \gamma_{1}^{\prime}\right)
$$


where $\Gamma_{0}$ and $\Gamma_{1}=\Gamma_{11} \times \Gamma_{12} \ldots \times \Gamma_{1 k}$ is strict subsets of the support of $x_{t}$. A standard two-step approach based on concentration and grid search can be used to compute the estimates. First, for each $\gamma_{0}$, we vary $\gamma_{1} \in \Gamma_{1}$ and compute the sum-of-squared errors $\widetilde{S S R}\left(\gamma_{0}, \gamma_{1}^{\prime}\right)$. Second, we find the minimum $\widetilde{S S R}_{T}\left(\gamma_{0}, \tilde{\gamma}_{1}\right)$, and then the least-squares estimates $\left(\hat{\gamma}_{0}, \hat{\gamma}_{1}^{\prime}\right)$ are the values which jointly minimize $\widetilde{S S R}_{T}\left(\gamma_{0}, \tilde{\gamma}_{1}\right)$. As suggested by Hansen (1999), it is undesirable for selecting the parameters $\left(\hat{\gamma}_{0}, \hat{\gamma}_{1}^{\prime}\right)$ that sort too few observations into one or the other regime. In practice, we can exclude this possibility by restricting the grid search in (5) to values of $\left(\gamma_{0}, \gamma_{1}^{\prime}\right)$ such that a minimal percentage of the observations (say, $5 \%$ or $10 \%$ ) lie in both regimes.

We have also developed test statistics for threshold effect $\left(F_{1 C}\right.$ and $\left.F_{1 T}\right)$ and threshold constancy $\left(F_{2}\right)$, and proposed to construct confidence intervals for the threshold coefficients by test inversion. See Appendix B for the details.

\section{Empirical Investigation}

In this section, we investigate whether the effect of debt on growth can be adequately characterized using Hansen's RK model with constant threshold or if the effect is better described by the modified model proposed in the previous section where a time-varying threshold is allowed. As emphasized by Panizza and Presbitero (2013), net public debt is difficult to compute and hardly comparable across countries. We therefore, following Lin (2014) and Hansen (2017), take a time-series approach, focusing on a single country. ${ }^{6}$ The data we use are the same as in Hansen (2017), available from Hansen's website. ${ }^{7}$ The data set covers annual observations of debt, GDP and inflation over 1791-2009, from the U.S. Specifically, we consider the following RK model with a state-dependent threshold

$$
y_{t}=\beta_{1}\left(x_{t}-\gamma_{t}\right)_{-}+\beta_{2}\left(x_{t}-\gamma_{t}\right)_{+}+\beta_{3} y_{t-1}+\beta_{0}+e_{t}
$$

where $y_{t}$ is the GDP growth rate in year $t$ and $x_{t}$ is the debt to GDP ratio in year $t-1$. Following Hansen (2017), the lagged dependent variable $y_{t-1}$ is added as an independent variable to ensure that the errors $e_{t}$ are approximately serially uncorrelated.

The debt-threshold (or tipping point) $\gamma_{t}$ is specified as a function of informative covariates. In this paper, three likely macroeconomic variables — lagged growth, in-

\footnotetext{
${ }^{6}$ By focusing on a single country, we are able to avert the complications of cross-country heterogeneity and error cross-section dependence that prevail in cross-country panel regressions. See Chudik et al. (2017) for a recent paper on the treatment of these complications.

${ }^{7}$ https://www.ssc.wisc.edu/ bhansen/
} 
flation, and past (debt/GDP) ratio — are considered. ${ }^{8}$ The rationale of considering these variables is laid out in Section 1. In choosing among these three threshold determinants, we employ a stepwise approach. We start with estimating three models (each model is with one of the three covariates) and choose the best one. Then, we examine if adding other covariates (one by one) in the best chosen model may make improvement. We report the modelling results in Tables 1 and 2 , in which $p$-values are calculated with $B=2000$ bootstrap replications.

\begin{tabular}{|c|c|c|c|c|c|c|c|c|c|}
\hline & & \multicolumn{2}{|c|}{$\begin{array}{c}\text { Constant threshold } \\
\text { Hansen's model }\end{array}$} & \multicolumn{2}{|c|}{ Growtht-1 } & \multicolumn{2}{|c|}{ Inflationt } & \multicolumn{2}{|r|}{ Debt $t-2$} \\
\hline \multirow[t]{3}{*}{ Slopes } & $\beta_{1}$ & 0.033 & {$[-0.002,0.136]$} & 0.072 & {$[-0.051,0.198]$} & 0.063 & {$[0.007,0.117]$} & 0.049 & {$[-0.082,0.179]$} \\
\hline & $\beta_{2}$ & -0.067 & {$[-0.178,-0.006]$} & -0.035 & {$[-0.099,0.025]$} & -0.068 & {$[-0.126,-0.011]$} & -0.206 & {$[-0.379,-0.033]$} \\
\hline & $\beta_{3}$ & 0.279 & {$[0.14,0.43]$} & 0.315 & {$[0.144,0.487]$} & 0.248 & {$[0.110,0.391]$} & 0.291 & {$[0.143,0.438]$} \\
\hline Intercept & $\beta_{0}$ & 3.782 & {$[2.58,4.94]$} & 3.643 & {$[2.291,5.025]$} & 4.831 & {$[3.660,6.003]$} & 3.345 & {$[2.163,4.525]$} \\
\hline \multirow[t]{2}{*}{ Threshold } & Constant & 43.8 & {$[30.8,70]$} & 16 & {$[10,55]$} & 44 & {$[13,66]$} & 16 & {$[10,64]$} \\
\hline & Growtht-1 & & & 1.9 & {$[-6.5,5,8]$} & & & & \\
\hline \multirow{3}{*}{ Testing } & $F_{1 C}$ & 5.655 & 0.150 & & & & & & \\
\hline & $F_{1 T}$ & & & 6.367 & 0.306 & 17.655 & 0.022 & 7.031 & 0.179 \\
\hline & $F_{2}$ & & & 0.666 & 0.631 & 11.618 & 0.027 & 1.544 & 0.381 \\
\hline
\end{tabular}

In Table 1, among the three models with a time-varying threshold, clearly the model with inflation outperforms the others (in terms of the $R$-squared). According to the $F_{1 T}$ test statistic, the model with inflation is the only model that can reject the null hypothesis of linearity $(p$-value $=0.022)$. None of the other nonlinear models (including the constant threshold model of Hansen (2017), replicated and reported in column 1 of Table 1 ) is able to beat the linear model. Meanwhile, according to the $F_{2}$ test statistic, the model with inflation is the only model that can reject the null hypothesis of constant threshold ( $p$-value $=0.027)$. For the model with inflation, the estimated inflation coefficient is -3.5 and the $90 \%$ confidence interval is $(-6.7,-0.2)$; therefore, the effect of inflation to the threshold is negative and statistically significant. We note that a negative inflation effect seems to accord well with the claim of Cochrane (2011a, b). As for growth and past debt, while the estimated coefficients of these two variables are with the expected sign, they are insignificant.

In Table 2, we report the results from specifying the threshold as a function of inflation and growth or the past debt ratio. In these two models, both the null hypothesis

\footnotetext{
${ }^{8}$ As suggested by Cochrane (2011a, b), debt could lead to an increase in expected inflation, hence we choose the inflation in year $t$.
} 
Table 2

Estimates based on candidate pairs (1791-2009) and bootstrap 90\% confidence intervals.

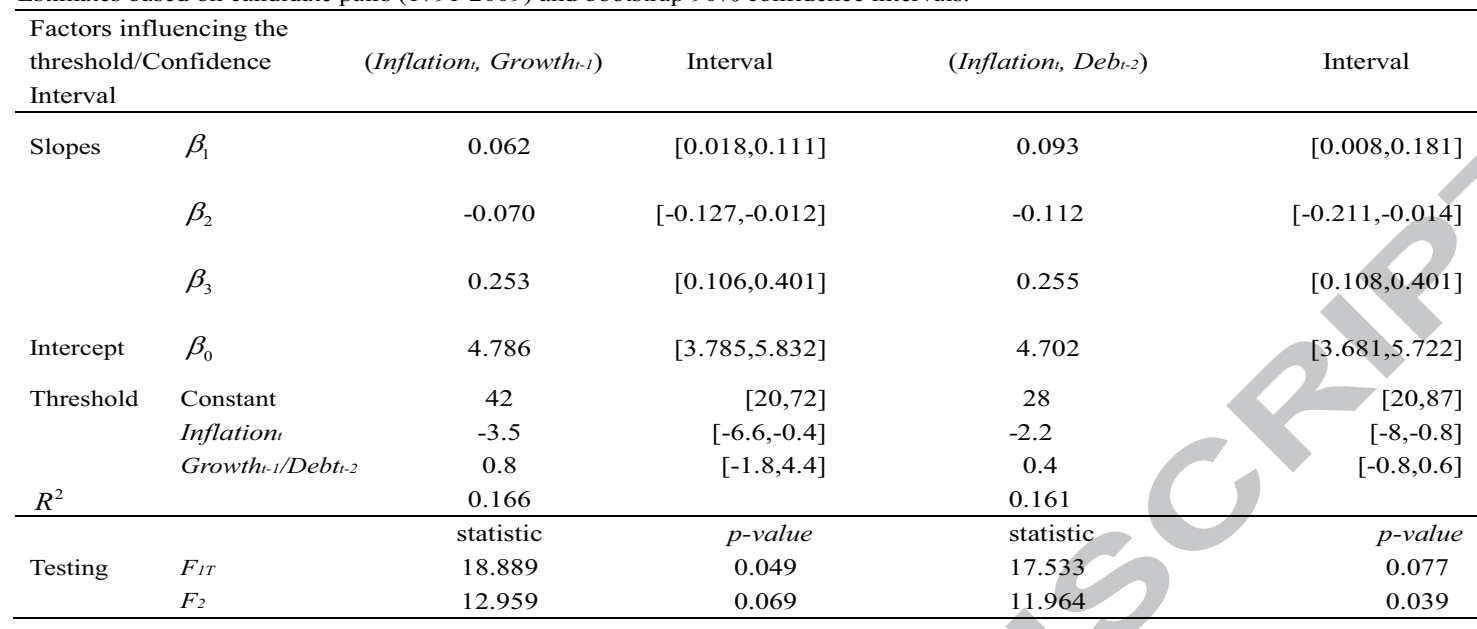

of linearity and the null hypothesis of constant threshold are rejected (according to $F_{1 T}$ and $F_{2}$, respectively). However, adding growth or past debt seems to contribute very little to the modelling outcome. From Tables 1 and 2, the model with a single covariate (inflation) appears to be the best among all considered time-varying threshold models. Comparing Hansen's (2017) results with those from the best time-varying threshold model, we find similar negative debt effects on growth when the debt-to-GDP ratio is above the threshold, regardless the threshold is constant or state-dependent. However, interestingly, when debt/GDP is below the threshold, while both models show positive debt effects the magnitude of the effect of our time-varying model is nearly double that of the Hansen model. Moreover, the positive debt effect is statistically significant in our model, but not in Hansen's.

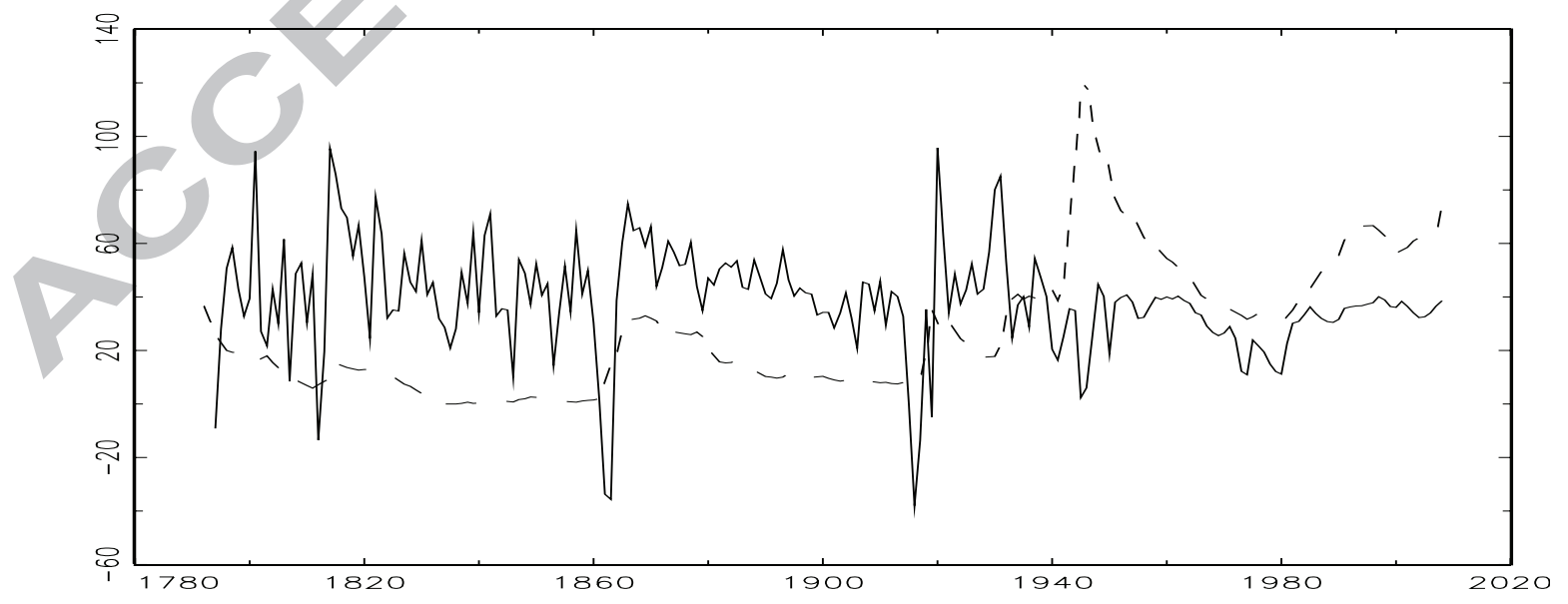

Fig. 1. The actual debt/GDP ratio (dashed line) and the estimated time-varying threshold (solid

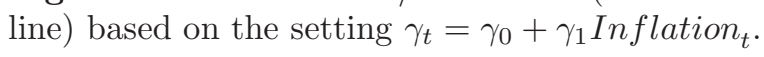

To take a further look at the time-varying threshold of the model with inflation, 
we depict the estimated threshold in Figure 1. The resulting threshold in Figure 1 is very volatile and a threshold oscillating from year to year may not be desirable for policy-guidance. ${ }^{9}$ Therefore, we next consider to specify the threshold as a function of the average of growth/inflation/debt over the past 5 years — denoted as $\overline{\mathrm{Growth}}_{5, t}$ $\overline{\text { Inflation }}_{5, t}$ and $\overline{\operatorname{Debt}}_{5, t-2}$. Similarly, we also employ a stepwise modelling strategy and report the empirical results in Tables 3 and 4.

Table 3

Estimates based on the averages over the past 5 years (1791-2009) and bootstrap 90\% confidence intervals.

Factors influencing the

threshold/Confidence Interval

$\overline{\text { Growth }}_{5,-1}$

Interval

$\overline{\text { Inflation }}_{5}$

Interval

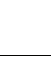

$\overline{D e b t}_{5, t-2}$

Interval

Slopes $\quad \beta$

$\beta_{2}$

$-0.076 \quad[-0.237,0.088]$

0.015

$[-0.052,0.083]$

$-0.012 \quad[-0.072,0.045] \quad-0.041$

$[-0.122,0.039]$

0.132

$[0.012,0.252]$

$\beta_{3}$

0.352

$[0.195,0.509]$

0.260

$[0.115,0.405]>0.270$

$[-0.237,-0.046]$

Intercept $\quad \beta_{0}$

Threshold Constan

2.543

$[1.203,3.882]$

3.765

$[2.368,5.163]$

3.848

$\overline{\text { Inflation }} / \overline{\text { Growth }} / \overline{\mathrm{Deb} t}$

$42 \quad[18,65]$

$55 \quad[13,69] \quad 10$

$[-12,2.9] \quad-8.9 \longrightarrow 0.8$

$[2.739,4.958]$

\begin{tabular}{llr}
$R^{2}$ & & 0. \\
\hline \multirow{3}{*}{ Testing } & & stati \\
& $F_{I T}$ & 7.1 \\
& $F_{2}$ & 2.202 \\
\hline
\end{tabular}

$\begin{array}{lccccc}0.116 & 0.118 & & 0.127 & \\ \text { tistic } & p \text {-value } & \text { statistic } & p \text {-value } & \text { statistic } & p \text {-value } \\ 127 & 0.318 & 7.688 & 0.165 & 10.065 & 0.095 \\ 202 & 0.419 & 2.736 & 0.287 & 4.998 & 0.072\end{array}$

According to Tables 3 and 4, the model with a single covariate of past debt appears to outperform the others - in this model, both $F_{1 T}$ and $F_{2}$ are with the $p$-value less than $10 \%$, indicating the existence of a time-varying threshold effect. This significance of past debt is generally in agreement with the view that the perceived "normal" debt level (threshold) may shift toward to the average debt level over the past few years. Interestingly, the estimated negative debt effect on growth appears to be relatively stronger when the model with average debt in the past is considered (Column 1, Table 3). We depict the past debt driven threshold in Figure 2 - clearly, the threshold is much less volatile than the inflation driven threshold in Figure 1. On the other hand, growth and inflation do not seem to contribute much to the threshold function. ${ }^{10}$

The previous analysis shows that inflation in year $t$ and the past 5-year debt average are two important factors influencing the threshold. We therefore consider the case where the threshold is a function of these two variables and report the modelling results in Table 5. This model appears to perform well: linearity and constancy are rejected and the coefficients of both factors are with the expected sign and significant. We depict the result threshold in Figure 3.

Overall, our results based on the time-varying threshold model support the existence

\footnotetext{
${ }^{9}$ We thank the anonymous referee to raise this point to us.

${ }^{10}$ The result might be partly due to the "over-smoothness" of the 5 -year average of growth/inflation.
} 
Table 4

Estimates based on candidate pairs of the averages (1791-2009) and bootstrap 90\% confidence intervals.

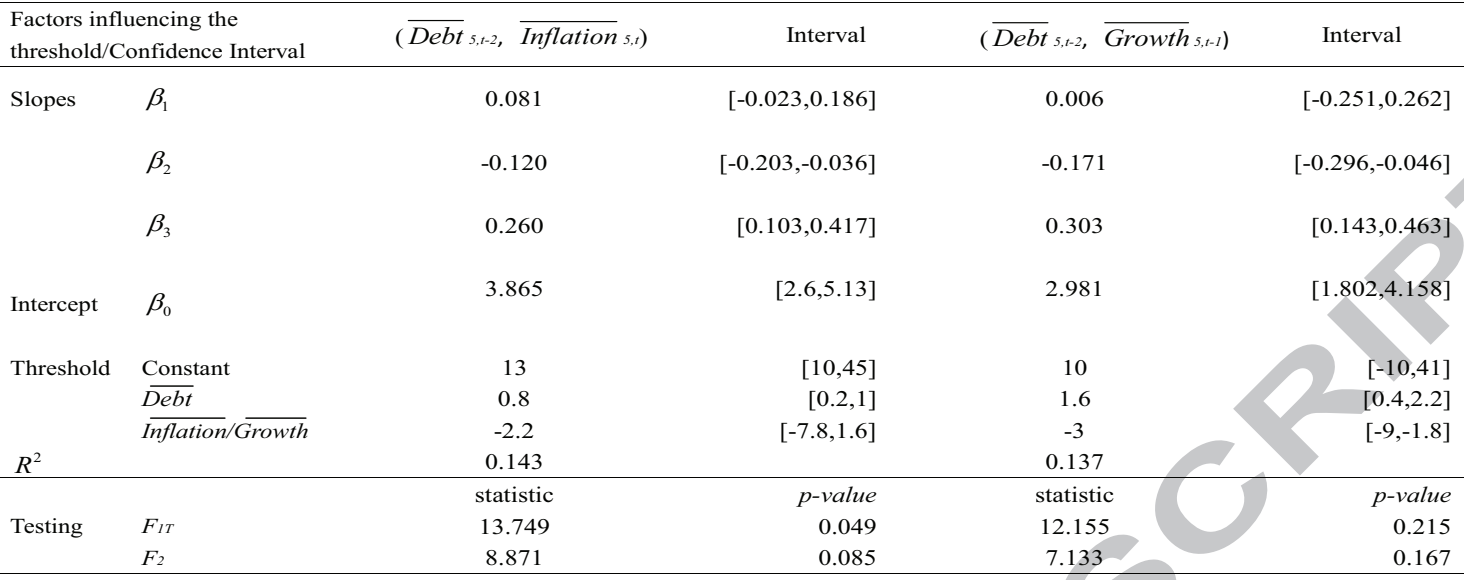

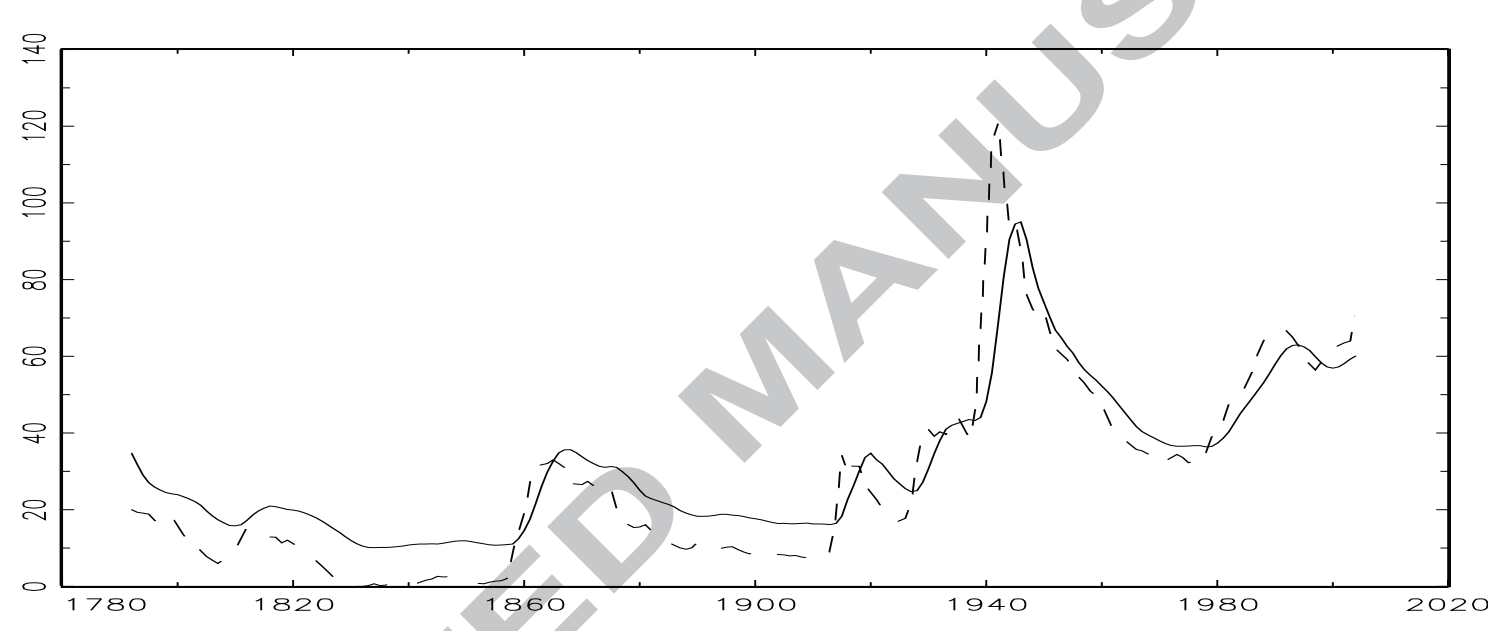

Fig. 2. The actual debt/GDP ratio (dashed line) and the estimated time-varying threshold (solid line) based on the setting $\gamma_{t}=\gamma_{0}+\gamma_{1} \overline{D e b t}_{5, t-2}$.

Table 5

Estimates based on inflation in year $t$ and 5-year average of debt (1791-2009) and bootstrap 90\% confidence intervals.

\begin{tabular}{|c|c|c|c|}
\hline & - & $\gamma_{t}=\gamma_{0}+\gamma_{1} \overline{\operatorname{Debt}}_{5, t-2}+\gamma_{2}$ Inflation $_{t}$ & Interval \\
\hline \multirow[t]{3}{*}{ Slopes } & $\beta_{1}$ & 0.075 & {$[0.001,0.191]$} \\
\hline & $\beta_{2}$ & -0.132 & {$[-0.226,-0.048]$} \\
\hline & $\beta_{3}$ & 0.248 & {$[0.113,0.394]$} \\
\hline Intercept & $\beta_{0}$ & 4.256 & {$[3.320,5.291]$} \\
\hline \multirow[t]{3}{*}{ Threshold } & Constant & 26 & {$[10,57]$} \\
\hline & $\overline{D e b t}$ & 0.7 & {$[0.2,1]$} \\
\hline & Inflation & -1.9 & {$[-4.8,-0.2]$} \\
\hline$R^{2}$ & & 0.155 & \\
\hline \multirow{3}{*}{ Testing } & & statistic & p-value \\
\hline & $\mathrm{F}_{2}$ & 16.748 & 0.039 \\
\hline & & 11.879 & 0.033 \\
\hline
\end{tabular}




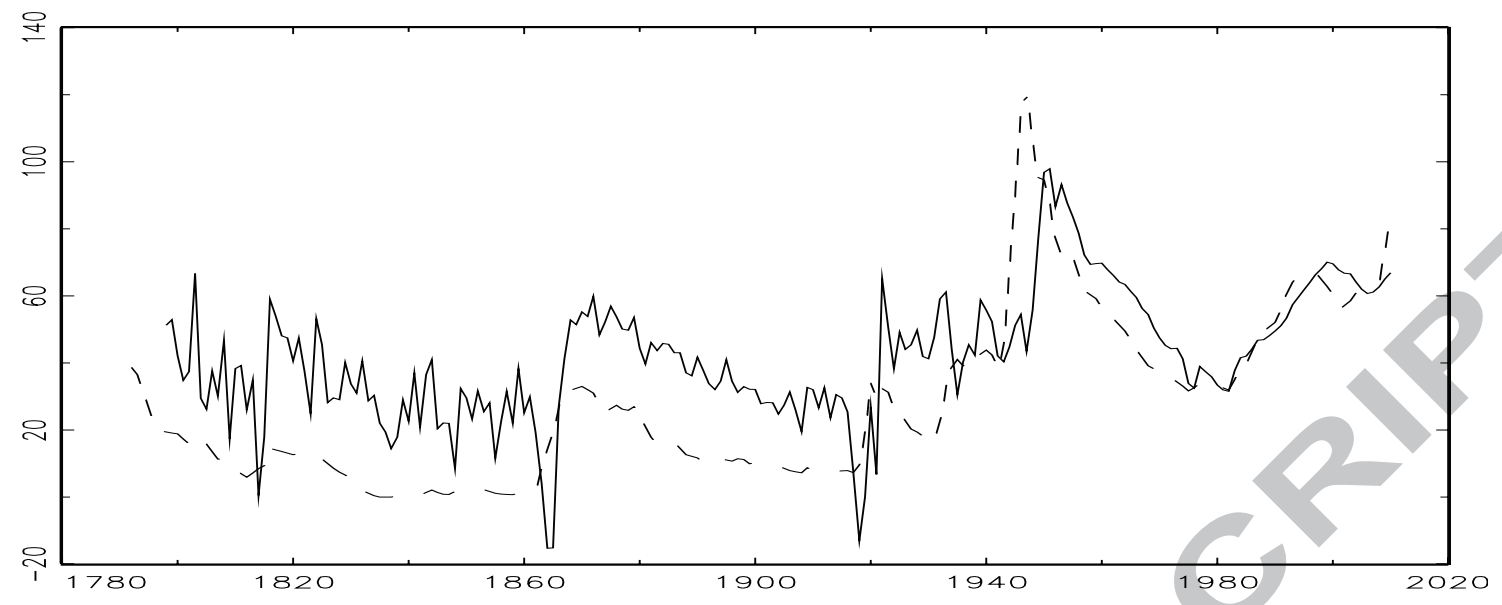

Fig. 3. The actual debt/GDP ratio (dashed line) and the estimated time-varying threshold (solid line) based on the setting $\gamma_{t}=\gamma_{0}+\gamma_{1} \overline{\text { Debt }}_{5, t-2}+\gamma_{2} \overline{\text { Inflation }}_{t}$.

of a debt-threshold effect, above which debt becomes notably harmful for growth. Moreover, we find clear evidence supporting that the debt-threshold is time-varying and state-dependent, and overlooking such time-varying and state-dependent features in the threshold might lead to very different results.

\section{Robustness of Results}

To further check the consistency of our findings regarding debt-threshold and its constancy, we run the RK models with a 100-year window moving up by 10 years. In each case, there are 13 rolling results, staring from 1792-1891 and ending in 1912-2009.

As a benchmark, we first estimate the constant threshold model of Hansen (2017) and report the results in Panel A of Table 6. It can be easily seen that the null of linearity can be rejected in only three sub-periods (1842-1941, 1872-1981 and 18821991) at the $10 \%$ significance level. This is generally consistent with the full-sample results.

We then turn to the time-varying model. Based on the findings in the previous section, two covariates - inflation and the average of 5-year debt/GDP ratios - are considered. We report the results in Panel B of Table 6. The rolling results show that 8 out of the 13 sub-periods have a threshold effect and the threshold is state-dependent. Interestingly, these 8 sub-periods are the last 8 sub-periods of the full sample. Overall, both our full-sample and sub-sample results support that there is a threshold effect of debt on growth and the threshold is better to be characterised in a time-varying fashion. 
Table 6

Estimates based on rolling subsamples and the constant/time-varying threshold specifications Panel A: Estimates based the constant threshold model developed by Hansen (2017)

\begin{tabular}{|c|c|c|c|c|c|c|c|c|c|c|c|c|c|c|}
\hline & & $1792-1891$ & $1802-1901$ & $1812-1911$ & $1822-1921$ & $1832-1931$ & $1842-1941$ & $1852-1951$ & $1862-1961$ & $1872-1971$ & $1882-1981$ & $1892-1991$ & $1902-2001$ & 1912-2009 \\
\hline \multirow[t]{3}{*}{ Slopes } & $\beta_{1}$ & -0.028 & 0.900 & -0.141 & 1.565 & -0.183 & -0.143 & 0.082 & 0.111 & 0.117 & 0.102 & 0.099 & 0.111 & 0.127 \\
\hline & $\beta_{2}$ & 0.259 & -0.037 & 0.043 & -0.033 & 0.039 & 0.272 & -0.051 & -0.097 & -0.096 & -0.087 & -0.077 & -0.075 & -0.073 \\
\hline & $\beta_{3}$ & 0.402 & 0.206 & 0.124 & 0.028 & 0.021 & 0.158 & 0.272 & 0.022 & 0.207 & 0.197 & 0.201 & 0.234 & 0.319 \\
\hline Intercept & $\beta_{0}$ & 2.116 & 3.905 & 3.042 & 4.406 & 3.117 & 1.131 & 4.329 & 5.750 & 5.720 & 5.181 & 4.731 & 4.527 & 3.978 \\
\hline \multirow[t]{3}{*}{ Threshold } & constant & 25.100 & 1.960 & 7.400 & 1.260 & 7.200 & 22.000 & 33.200 & 43.800 & 43.200 & 44.700 & 44.100 & 43.860 & 42.400 \\
\hline & $F I C$ & 3.115 & 0.754 & 0.721 & 0.375 & 1.209 & 7.676 & 5.560 & 6.253 & 8.117 & 7.023 & 6.538 & 7.686 & 7.893 \\
\hline & $p$-value & 0.218 & 0.411 & 0.682 & 0.546 & 0.671 & 0.018 & 0.146 & 0.150 & 0.080 & 0.099 & 0.153 & 0.123 & 0.121 \\
\hline \multicolumn{15}{|c|}{ Panel B: Estimates based on the setting where the threshold is a function of inflation of a single year and the average of 5-year debt/GDP ratios } \\
\hline \multirow{4}{*}{ Slopes } & & $1792-1891$ & $1802-1901$ & $1812-1911$ & $1822-1921$ & $1832-1931$ & $1842-1941$ & $1852-1951$ & $1862-1961$ & $1872-1971$ & $1882-1981$ & $1892-1991$ & $1902-2001$ & 1912-2009 \\
\hline & $\beta_{1}$ & 0.183 & 0.199 & 0.251 & 0.226 & 0.361 & 0.214 & 0.186 & 0.267 & 0.293 & 0.229 & 0.171 & 0.149 & 0.253 \\
\hline & $\beta_{2}$ & -0.519 & -0.574 & -0.632 & -0.571 & -0.346 & -0.198 & -0.212 & -0.281 & & -0.196 & -0.169 & -0.151 & -0.091 \\
\hline & $\beta_{3}$ & 0.327 & 0.211 & 0.109 & 0.015 & 0.099 & 0.089 & 0.057 & & 0.064 & 0.068 & 0.064 & 0.108 & 0.218 \\
\hline Intercept & $\beta_{0}$ & 4.525 & 5.058 & 5.756 & 5.932 & 6.445 & 9.149 & 11.096 & 10.872 & 9.866 & 7.839 & 6.996 & 6.368 & 5.179 \\
\hline \multirow[t]{4}{*}{ Threshold } & constant & 11 & 11 & 11 & 10 & 10 & 33 & 52 & 32 & 26 & 27 & 37 & 40 & 33 \\
\hline & $\overline{d e b t}$ & 0.6 & 0.6 & 0.6 & 0.7 & 0.8 & 0.5 & 0.3 & 0.8 & 0.8 & 0.8 & 0.7 & 0.7 & 0.2 \\
\hline & Inflation & -0.4 & -0.3 & -0.3 & -0.1 & -0.7 & -2.7 & -3.6 & -2.6 & -2.5 & -2.8 & -3.5 & -3.9 & -2.7 \\
\hline & $F_{I T}$ & 9.813 & 6.596 & 6.359 & 7.675 & 9.222 & 22.512 & 42.298 & 42.914 & 40.651 & 32.970 & 29.959 & 30.746 & 30.981 \\
\hline \multirow[t]{3}{*}{$\mathrm{p}$-values } & $p$-value & 0.283 & 0.350 & 0.326 & 0.266 & 0.202 & 0.010 & 0.000 & 0.000 & 0.000 & 0.003 & 0.006 & 0.023 & 0.033 \\
\hline & $F_{2}$ & 7.315 & 4.831 & 5.285 & 6.362 & 7.899 & 13.766 & 34.933 & 34.933 & 34.500 & 27.815 & 18.668 & 17.030 & 21.323 \\
\hline & $p$-value & 0.227 & 0.237 & 0.137 & 0.177 & 0.093 & 0.007 & 0.000 & 0.000 & 0.000 & 0.000 & 0.011 & 0.013 & 0.040 \\
\hline
\end{tabular}

\section{Conclusion}

Despite extensive research which has already been undertaken, the issue as to whether there is a debt-threshold effect, continues to be strongly debated. We highlight the crucial role of the time-varying and state-dependent threshold in this debate. The contribution of this paper is two-fold. First, we contribute to the econometric literature by extending the regression kink model of Hansen (2017) to allow for a time-varying, state-dependent threshold. Through Monte Carlo simulations, we show that overlooking possible time-varying features of a threshold often ends up with biased estimates. Second, we contribute to the debt-growth literature by applying our model to the U.S. historical data. Our results show that there is evidence supporting a nonlinear effect of debt on growth. Moreover, we find clear evidence supporting that the debt/GDP threshold is time-varying and state-dependent. In general, our results are robust when rolling subsamples are considered.

One limitation of our paper is that the choice of debt-threshold determinants is arbitrary. It is imperative to develop a theory that may lead us toward a more sys- 
tematic approach on the choice. Also, similar to the analysis of Hansen (2017), an important caveat of our paper is that the analysis is based on a single time series of the United States, thus ignoring other countries' information. Future work could extend the time-series model to a panel setting by following the work of Chudik et al. (2017). 


\section{Appendix A: Monte Carlo Simulations}

In this section, through Monte Carlo simulations we compare the finite sample performances of the proposed estimation procedure with a time-varying/state-dependent threshold and Hansen's (2017) estimation procedure with an unknown constant threshold, and provide evidence to support that overlooking the time-varying features in the threshold can result in biased estimates.

The data generating process is specified as

$$
y_{t}=\beta_{1}\left(x_{t}-\gamma_{t}\right)_{-}+\beta_{2}\left(x_{t}-\gamma_{t}\right)_{+}+\beta_{3} z_{t}+e_{t}, t=1,2, \ldots, T
$$

where $\gamma_{t}=\gamma_{0}+\gamma_{1} q_{1 t}+\gamma_{2} q_{2 t}$. We assume a normally distributed error $e_{t} \sim$ i.i.d.N $(0,1)$, $q_{1 t}, q_{2 t}$ and $z_{t} \sim$ i.i.d.N(0,1), $x_{t} \sim$ i.i.d.N $(0,4)$. The sample size $T$ is set as 50,100 or 200.

In the simulations, we set $\beta_{1}=1, \beta_{2}=2, \beta_{3}=1, \gamma_{0}=1$. We vary the parameters $\gamma_{1}$ and $\gamma_{2}$ to assess the models' performance to the existence of the time-varying features in the threshold. Specifically, we set $\left(\gamma_{1}, \gamma_{2}\right)=(1,2)$ or $(0,0)$. The number of replications is 1000 .

First, consider the performance of the proposed estimation procedure with a timevarying threshold. Table A.1 presents the summary statistics (i.e., mean, median, and standard deviation) for the estimates. From the summary statistics (i.e., mean and standard deviation) reported in Table A.1, the proposed estimates seem to be unbiased and the accuracy of the model improves as $T$ increases. Overall, for the small sample sizes the performance of the proposed estimation procedure is generally satisfactory.

Table A.1

Estimates of the parameters using the procedure proposed in Section 2.

\begin{tabular}{|c|c|c|c|c|c|c|c|c|c|c|c|c|}
\hline \multirow{3}{*}{$\frac{\left(\gamma_{1}, \gamma_{2}\right)}{(1,2)}$} & \multicolumn{6}{|c|}{ threshold } & \multicolumn{6}{|c|}{ coefficients } \\
\hline & \multicolumn{2}{|c|}{$\gamma_{0}=1$} & \multicolumn{2}{|c|}{$\gamma_{1}=1$} & \multicolumn{2}{|c|}{$\gamma_{2}=2$} & \multicolumn{2}{|c|}{$\beta_{1}=1$} & \multicolumn{2}{|c|}{$\beta_{2}=2$} & \multicolumn{2}{|c|}{$\beta_{3}=1$} \\
\hline & Mean & Std.dev & Mean & Std.dev & Mean & Std.dev & Mean & Std.dev & Mean & Std.dev & Mean & Std.dev \\
\hline $\mathrm{T}=50$ & 0.992 & 0.796 & 0.992 & 0.158 & 2.011 & 0.177 & 0.985 & 0.151 & 2.032 & 0.234 & 0.987 & 0.146 \\
\hline $\mathrm{T}=100$ & 0.997 & 0.552 & 1.013 & 0.111 & 2.006 & 0.145 & 0.976 & 0.086 & 2.025 & 0.164 & 0.994 & 0.101 \\
\hline $\mathrm{T}=200$ & 1.003 & 0.301 & 0.997 & 0.085 & 2.011 & 0.088 & 0.992 & 0.060 & 2.000 & 0.098 & 1.011 & 0.063 \\
\hline \multicolumn{13}{|l|}{$(0,0)$} \\
\hline $\mathrm{T}=50$ & 0.992 & 0.883 & 0.023 & 0.180 & 0.006 & 0.150 & 0.943 & 0.245 & 2.110 & 0.540 & 1.002 & 0.142 \\
\hline $\mathrm{T}=100$ & 1.005 & 0.513 & 0.020 & 0.108 & -0.003 & 0.115 & 0.991 & 0.091 & 2.065 & 0.259 & 1.004 & 0.097 \\
\hline $\mathrm{T}=200$ & 1.002 & 0.329 & -0.003 & 0.075 & 0.005 & 0.088 & 1.003 & 0.066 & 2.011 & 0.162 & 0.998 & 0.078 \\
\hline
\end{tabular}

Notes: Std.dev denotes standard deviation. The simulations were written in the GAUSS programming language. 
Next, consider the case where the data generating process is given by (A1), while the time-varying feature is overlooked in the estimation procedure (and hence the estimation procedure proposed by Hansen's (2017) is used). Table A.2 presents the summary statistics (i.e., mean and standard deviation) for the parameter estimates. Clearly, the estimation procedure proposed by Hansen (2017) works reasonably well when the data generating process does not contain time-varying thresholds (i.e. $\gamma_{1}=$ $\gamma_{2}=0$ ). However, the results show that, if the time-varying feature in threshold is ignored, the estimates are seriously biased and with large standard errors. Our unreported simulations also show that the direction and the magnitude of the bias depend on the time-varying threshold parameters.

Furthermore, the results from Table A.1 shows that there is little efficiency loss using the proposed time-varying threshold estimation procedure when the data generating process is without such time-varying features in the threshold. Combining the results of Tables A.1 and A.2, we conclude that the proposed varying threshold estimation procedure is more robust to misspecification of the threshold, especially when the researcher does not have enough confidence in the threshold constancy.

Table A.2

Estimates of the parameters based on the Hansen's (2017) constant threshold procedure.

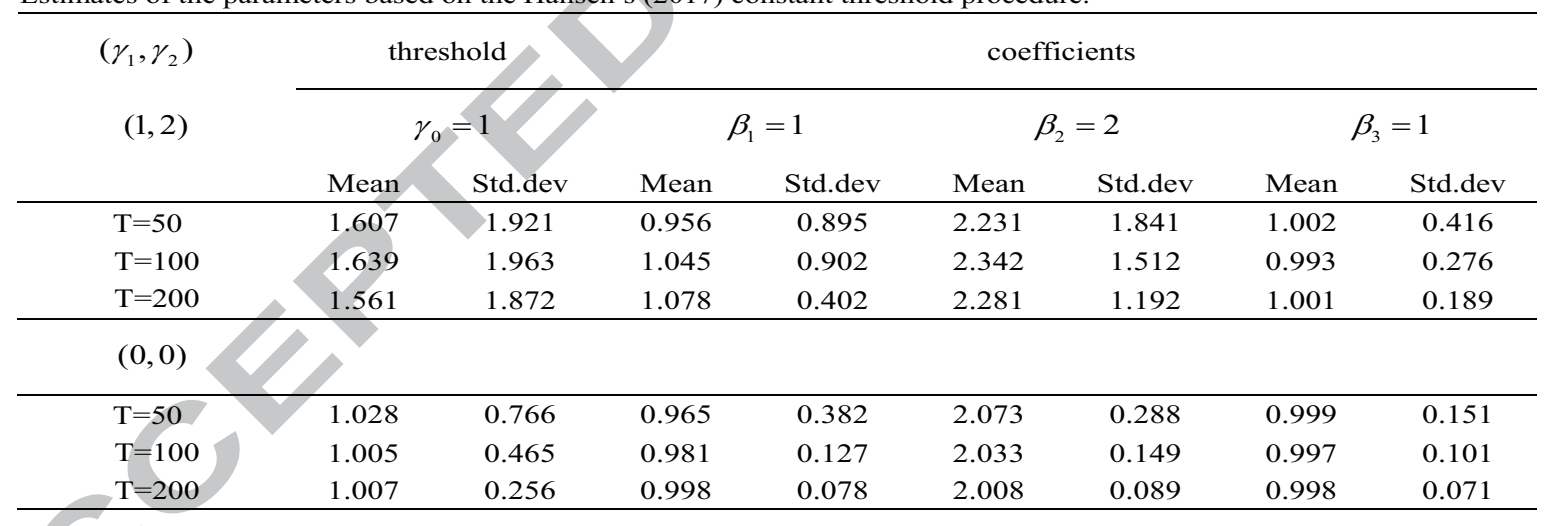

Notes: Std.dev denotes standard deviation. The simulations were written in the GAUSS programming language.

\section{Appendix B: Test Statistics}

To determine whether the RK model (1) with a threshold is significantly different from the linear model $y_{t}=\beta_{1} x_{t}+\boldsymbol{\beta}_{3}^{\prime} \boldsymbol{z}_{t}+e_{t}$, which is nested in the RK model (1), we consider to test the hypothesis of no threshold effect in the RK model (1)

$$
H_{0}^{1}: \beta_{1}=\beta_{2}
$$


The linear model $y_{t}=\beta_{1} x_{t}+\beta_{3}^{\prime} z_{t}+e_{t}$ can be estimated by OLS, yielding the estimates $\hat{\beta}_{1}, \hat{\beta}_{3}$, residuals $\hat{e}_{t}$ and the sum of squared errors $S S R_{0}=\sum_{t=1}^{T} \hat{e}_{t}^{2}$. Likewise, we denote $S S R_{C}$ as the sum of squared errors from the constant threshold model of Hansen (2017). A standard test for the null hypothesis of the linear model against the constant threshold model in Hansen $(2017)$ is $F_{1 C}$

$$
F_{1 C}=\frac{S S R_{0}-S S R_{C}}{S S R_{C} / T} .
$$

Furthermore, a natural test for the null hypothesis of the linear model against the time-varying threshold model (1) is to reject $H_{0}^{1}: \beta_{1}=\beta_{2}$ for large values of the $F$-type statistic

$$
F_{1 T}=\frac{S S R_{0}-\widetilde{S S R}_{T}\left(\hat{\gamma}_{0}, \hat{\gamma}_{1}^{\prime}\right)}{\widetilde{S S R}_{T}\left(\hat{\gamma}_{0}, \hat{\gamma}_{1}^{\prime}\right) / T}
$$

Hence, the linear model $y_{t}=\beta_{1} x_{t}+\beta_{3}^{\prime} z_{t}+e_{t}$ is rejected if either $F_{1 C}$ or $F_{1 T}$ rejects the null $H_{0}^{1}: \beta_{1}=\beta_{2}$. Under $H_{0}^{1}$ the threshold is not identified, and hence the null distributions of the test statistics are non-standard due to the well-known Davies' problem (Davies, 1977; 1987) and can be typically explored by taking the supremum of all possible values of unidentified parameters (e.g. Davies, 1987; Andrews and Ploberger, 1994; Hansen, 1996). In general, the construction of critical values for these distributions of test statistics is non-trivial (Andrews and Ploberger, 1994) and the $p$-values (or critical values) of test statistics can be easily generated using simulations as shown by Hansen (1996). We therefore propose to use a parametric bootstrap procedure in the tradition of Hansen $(1996 ; 2017)$ to construct the $p$-values of the test statistics.

\section{Algorithm 1. Testing for threshold effects}

Step 1. Generate $T$ iid draws $u_{t}^{*}$ from the $N(0,1)$ distribution.

Step 2. Set $y_{1 t}^{*}=\hat{e}_{t} u_{t}^{*}$ where $\hat{e}_{t}$ are the ordinary least-square (OLS) residuals of the linear model $y_{t}=\beta_{1} x_{t}+\boldsymbol{\beta}_{3}^{\prime} \boldsymbol{z}_{t}+e_{t}$.

Step 3. Using the observations $\left\{y_{1 t}^{*}, x_{t}, \boldsymbol{z}_{t}^{\prime}\right\}_{t=1}^{T}$, estimate the linear model, the Hansen's (2017) RK model with a constant threshold and the time-varying threshold model (1), and compute the F-type statistics $F_{1 C}$ and $F_{1 T}$.

Step 4. Repeat Steps 1-3 $B$ times, so as to obtain two samples $F_{1 C}^{*}(1), F_{1 C}^{*}(2), \ldots, F_{1 C}^{*}(B)$ and $F_{1 T}^{*}(1), F_{1 T}^{*}(2), \ldots, F_{1 T}^{*}(B)$ of simulated $F_{1 C}$ and $F_{1 T}$ statistics, respectively.

Step 5. The empirical $p$-values can be obtained by calculating the percentage of the 
simulated statistics that exceed actual value:

$$
\begin{aligned}
p_{1 C} & =\frac{1}{B} \sum_{b=1}^{B} 1\left(F_{1 C}^{*}(b) \geq F_{1 C}\right), \\
p_{1 T} & =\frac{1}{B} \sum_{b=1}^{B} 1\left(F_{1 T}^{*}(b) \geq F_{1 T}\right) .
\end{aligned}
$$

Step 6 . When necessary, compute the level $\alpha$ critical value $c_{\alpha}$ as the empirical $1-\alpha$ quantile of the simulated F-type statistics.

Another important problem is to determine whether or not the threshold is constant. To this end, consider the following null hypothesis

$$
H_{0}^{2}: \gamma_{1}=0
$$

We note that, under the null, the model in (1) is the RK model with an unknown constant threshold investigated by Hansen (2017). A standard test for the null hypothesis of the constant threshold against the time-varying threshold model (1) can be given by

$$
F_{2}=\frac{S S R_{C}-\widetilde{S S R}_{T}\left(\hat{\gamma}_{0}, \hat{\gamma}_{1}^{\prime}\right)}{\widetilde{S S R}_{T}\left(\hat{\gamma}_{0}, \hat{\gamma}_{1}^{\prime}\right) / T} .
$$

Furthermore, to construct confidence intervals for the threshold coefficients, we invert the following statistic for the null $H_{0}: \gamma=\gamma^{0}$ in which $\gamma=\left(\gamma_{0}, \gamma_{1}^{\prime}\right)^{\prime}$, given by

$$
F_{T}(\gamma)=\frac{\widetilde{S S R}_{T}(\gamma)-\widetilde{S S R}_{T}(\hat{\gamma})}{\widetilde{S S R}_{T}(\hat{\gamma}) / T}
$$

where $\hat{\gamma}=\left(\hat{\gamma}_{0}, \hat{\gamma}_{1}^{\prime}\right)^{\prime}$. The null hypothesis is rejected for large values of $F_{T}\left(\gamma^{0}\right)$. Following Hansen (2017), the $p$-value of $F_{2}$ and the confidence intervals of parameters can be computed by a model-based bootstrap method.

Algorithm 2. Testing for threshold constancy and computing confidence intervals for parameters

Step 1. Generate $T$ iid draws $u_{t}^{*}$ from the $N(0,1)$ distribution.

Step 2. Set $y_{2 t}^{*}=\hat{\boldsymbol{\beta}}^{\prime}(\hat{\gamma}) \boldsymbol{x}_{t}(\hat{\gamma})+\hat{e}_{t}(\hat{\gamma}) u_{t}^{*}$ where $(\hat{\boldsymbol{\beta}}(\hat{\gamma}), \hat{\gamma})$ and $\hat{e}_{t}(\hat{\gamma})$ are the parameter estimates and residuals of the Hansen's (2017) regression kink model with a constant threshold. 
Step 3. Using the observations $\left\{y_{2 t}^{*}, x_{t}, \boldsymbol{z}_{t}^{\prime}, \boldsymbol{q}_{t}^{\prime}\right\}_{t=1}^{T}$, estimate the Hansen's (2017) RK model with a constant threshold and the proposed time-varying threshold model (1), and compute the F-type statistic $F_{2}$.

Step 4. Repeat Steps 1-3 $B$ times, so as to obtain one sample $F_{2}^{*}(1), F_{2}^{*}(2), \ldots, F_{2}^{*}(B)$ of simulated $F_{2}$ statistics. The empirical $p$-value can be obtained by calculating the percentage of the simulated statistics that exceed actual value:

$$
p_{2}=\frac{1}{B} \sum_{b=1}^{B} 1\left(F_{2}^{*}(b) \geq F_{2}\right) .
$$

Step 5. Set $y_{3 t}^{*}=\hat{\boldsymbol{\beta}}^{\prime}(\hat{\gamma}) \boldsymbol{x}_{t}(\hat{\boldsymbol{\gamma}})+\hat{e}_{t}(\hat{\boldsymbol{\gamma}}) u_{t}^{*}$ where $(\hat{\boldsymbol{\beta}}, \hat{\gamma})$ and $\hat{e}_{t}(\hat{\boldsymbol{\gamma}})$ are the parameter estimates and residuals of the proposed regression kink model with a time-varying/statedependent threshold using the observations $\left\{y_{t}, x_{t}, \boldsymbol{z}_{t}^{\prime}, \boldsymbol{q}_{t}^{\prime}\right\}_{t=1}^{T}$.

Step 6. Using the observations $\left\{y_{3 t}^{*}, x_{t}, \boldsymbol{z}_{t}^{\prime}, \boldsymbol{q}_{t}^{\prime}\right\}_{t=1}^{T}$, estimate the regression kink model with a state-dependent threshold, yielding the parameter estimates $\left(\hat{\boldsymbol{\beta}}^{*}, \hat{\boldsymbol{\gamma}}^{*}\right)$, and the sum of squared errors $\widetilde{S S R}_{T}^{*}\left(\hat{\gamma}^{*}\right)$. Compute the $F$-statistic for $\hat{\gamma}$

$$
F_{T}^{*}(\hat{\gamma})=\frac{\widetilde{S S R}_{T}^{*}(\hat{\gamma})-\widetilde{S S R}_{T}^{*}\left(\hat{\gamma}^{*}\right)}{\widetilde{S S R}_{T}^{*}\left(\hat{\gamma}^{*}\right) / T}
$$

where $\widetilde{S S R}_{T}^{*}(\hat{\gamma})=\sum_{t=1}^{T}\left(y_{3 t}^{*}-\hat{\boldsymbol{\beta}}^{*^{\prime}}(\hat{\gamma}) \boldsymbol{x}_{t}(\hat{\gamma})\right)^{2}$.

Step 7. Repeat Steps 5-6 B times, and obtain a sample of simulated coefficient estimates $\left(\hat{\boldsymbol{\beta}}^{*}, \hat{\boldsymbol{\gamma}}^{*}\right)$ and $F$ statistic $F_{T}^{*}(\hat{\boldsymbol{\gamma}})$. Create $1-\alpha$ bootstrap confidence intervals for the slope parameters $\boldsymbol{\beta}=\left[\beta_{1}, \beta_{2}, \boldsymbol{\beta}_{3}^{\prime}\right]^{\prime}$ by the symmetric percentile method: the estimates plus and minus the $(1-\alpha)$ quantile of the absolute centered bootstrap estimates. For example, for $\beta_{1}$ the confidence interval is $\hat{\beta}_{1} \pm q_{1-\alpha}^{*}$, where $q_{1-\alpha}^{*}$ is the $1-\alpha$ quantile of $\left|\hat{\beta}_{1}^{*}-\hat{\beta}_{1}\right|$.

Step 8. Calculate the $1-\alpha$ quantile $c_{1-\alpha}^{*}$ of the simulated $F$ statistics $F_{T}^{*}$. Then we can create a $1-\alpha$ bootstrap confidence interval for $\gamma$ as follows:

$$
C_{\gamma}^{*}=\left\{\gamma: F_{T}(\gamma) \leq c_{1-\alpha}^{*}\right\}
$$




\section{References}

Andrews, D. W. K., Ploberger, W., 1994. Optimal tests when a nuisance parameter is present only under the alternative. Econometrica, 62(6), 1383-1414.

Baum, A., Checherita-Westphal, C., Rother, P., 2013. Debt and growth: New evidence for the euro area. Journal of International Money and Finance, 32, 809821.

Card, D., Lee, D. S., Pei, Z., Weber, A., 2017. Regression kink design: Theory and practice. In Regression Discontinuity Designs: Theory and Applications (Advances in Econometrics, Volume 38) Emerald Publishing Limited, pp.341 382

Cecchetti, S. G., Mohanty, M. S., Zampolli, F., 2011. The real effects of debt. BIS Working Papers No. 352.

Checherita-Westphal, C., Rother, P., 2012. The impact of high government debt on economic growth and its channels: An empirical investigation for the euro area. European Economic Review, 56(7), 1392-1405.

Chudik, A., Mohaddes, K., Pesaran, M. H., et al., 2017. Is there a debt-threshold effect on output growth? Review of Economics and Statistics, 99(1), 135-150.

Cochrane, J. H., 2011a. Inflation and debt. National affairs, 9(2), 56-78.

Cochrane, J. H., 2011b. Understanding policy in the great recession: Some unpleasant fiscal arithmetic. European Economic Review, 55(1): 2-30.

Dueker, M. J., Psaradakis, Z., Sola, M. and Spagnolo, F., 2013. State-Dependent Threshold Smooth Transition Autoregressive Models. Oxford Bulletin of Economics and Statistics, 75(6), 835-854.

Davies, R. B., 1977. Hypothesis testing when a nuisance parameter is present only under the alternative. Biometrika, 64(2), 247-254.

Davies, R. B., 1987. Hypothesis testing when a nuisance parameter is present only under the alternative. Biometrika, 74(1): 33-43.

Eberhardt, M., Presbitero, A. F., 2015. Public debt and growth: Heterogeneity and non-linearity. Journal of International Economics, 97(1), 45-58. 
Egert, B., 2015. Public debt, economic growth and nonlinear effects: Myth or reality?. Journal of Macroeconomics, 43, 226-238.

Hansen, B. E., 1996. Inference when a nuisance parameter is not identified under the null hypothesis. Econometrica, 64(2), 413-430.

Hansen, B.E., 2017. Regression Kink With an Unknown Threshold. Journal of Business and Economic Statistics, 35(2), 228-240.

Herndon, T., Ash, M., and Pollin, R., 2014. Does high public debt consistently stifle economic growth? A critique of Reinhart and Rogoff. Cambridge journal of economics, 38(2), 257-279.

Lin, T.-C., 2014. High-Dimensional Threshold Quantile Regression With an Application to Debt Overhang and Economic Growth. Working Paper, University of Wisconsin.

Lee S, Park H, Seo M H, et al., 2017. Testing for a Debt-Threshold Effect on Output Growth. Fiscal Studies, 38(4):701-717.

Panizza, U., Presbitero, A. F., 2013. Public debt and economic growth in advanced economies: A survey. Swiss Journal of Economics and Statistics, 149(2), 175-204. 


\section{Accepted Manuscript}

Debt and Growth: Is There a Constant Tipping Point?

Lixiong Yang, Jen-Je Su

PII:

S0261-5606(18)30365-6

DOI:

https://doi.org/10.1016/j.jimonfin.2018.06.002

Reference:

JIMF 1921

To appear in:

Journal of International Money and Finance

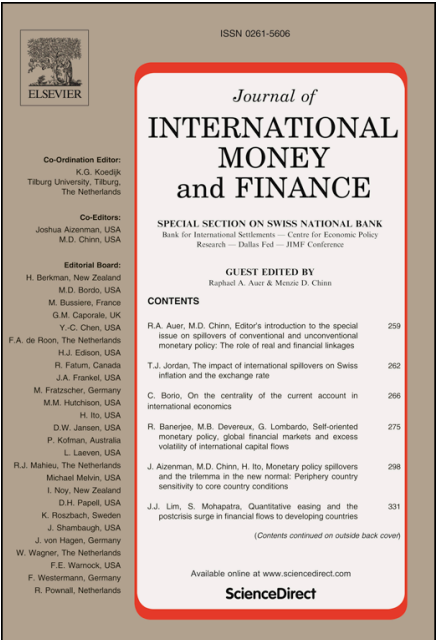

Please cite this article as: L. Yang, J-J. Su, Debt and Growth: Is There a Constant Tipping Point?, Journal of International Money and Finance (2018), doi: https://doi.org/10.1016/j.jimonfin.2018.06.002

This is a PDF file of an unedited manuscript that has been accepted for publication. As a service to our customers we are providing this early version of the manuscript. The manuscript will undergo copyediting, typesetting, and review of the resulting proof before it is published in its final form. Please note that during the production process errors may be discovered which could affect the content, and all legal disclaimers that apply to the journal pertain. 